\title{
Using an Unmanned Aircraft System (Drone) to Conduct a Complex High Altitude Search and Rescue Operation: A Case Study
}

\author{
Jake N. McRae, BSN; Christopher J. Gay, MPH; Brandon M. Nielsen, BS; Andrew P. Hunt, MBS \\ College of Osteopathic Medicine, Rocky Vista University, Ivins, UT
}

\begin{abstract}
Unmanned aircraft systems, also known as unmanned aerial vehicles or drones, are becoming increasingly common consumer products; their potential applications to search and rescue operations are becoming ever more apparent. A consumer drone was used to locate a mountaineer after he and his climbing partner were separated while summiting Broad Peak in the Karakoram Mountains of northeastern Pakistan, the world's 12th highest summit. The use of a commercial drone in the search for the missing climber allowed for a quick rescue mission, eliminating the need for a fully mobilized rescue party while significantly reducing the risk to the climbers charged with rescuing him. Without the drone, the rescue party would have faced immense challenges, including a vast search area and altitudes near the limits of human physiologic function. With real-time imagery and global positioning system capabilities, drones may become an invaluable tool in search and rescue operations, helping to reduce response time and maintain the safety of responders at high altitude and in many other types of difficult terrain.
\end{abstract}

Keywords: mountain rescue, unmanned aerial vehicle, first responder, wilderness medicine, SAR

\section{Introduction}

Government and military use of drones, a subclass of unmanned aerial vehicle (UAV), is relatively well tested, and drone use has become popular among the general public, with over 1 million drones registered with the Federal Aviation Administration (FAA). ${ }^{1}$ Many of the developments in drone use can be applied to search and rescue (SAR) operations. In emergency situations that require SAR, consumer and commercial drones have the potential to become a cost-effective, lifesaving tool for SAR personnel. ${ }^{2}$

In many environments, SAR teams send out large search parties to locate victims. This strategy has many added challenges when working at altitude, making serious attempts to locate victims more time intensive and dangerous for rescuers. These challenges have the potential to be mitigated with the use of drones. We present a case in which a drone was used to locate a lost climber

Corresponding author: Jake N. McRae, BSN, Rocky Vista University, College of Osteopathic Medicine, 250 Snow Canyon Dr., \#67, Ivins, UT 84738.; e-mail: jake.mcrae@ rvu.edu.

Submitted for publication October 2018.

Accepted for publication March 2019. and facilitate his rescue, obviating the need for a large search party that likely would have put more lives at risk.

\section{Case}

A 65-y-old Scottish mountaineer was found by using a Mavic Pro drone (DJI, Shenzhen, China) when he was stranded while attempting to descend Broad Peak in the Himalayas. ${ }^{3}$ While descending the west face of the mountain, the climber fell from an ice cliff, losing most of his gear in the process. ${ }^{4,5}$ Presuming him to be dead, his fellow climbers descended to a camp at a lower elevation without him. After reorienting from the fall, the climber found himself in a dangerous position on an extremely steep and icy aspect of the mountain. His crampons and ice tools were the only things keeping him from sliding further toward a cliff with a drop of several hundred meters. ${ }^{4}$ Furthermore, the situation was made more dangerous because he had no food or water.

On hearing that there was a lost climber on the mountain, another team of 2 Polish mountaineers and high altitude cinematographers used their DJI Mavic Pro, which was originally intended to be used for filming, in an attempt to locate him. ${ }^{4,5}$ The manufacturer of this specific 
drone claimed it has a range of $7 \mathrm{~km}$ and a battery life of $27 \mathrm{~min}^{3}{ }^{3}$ They flew the drone from the base camp of $\mathrm{K} 2$ to the victim's suspected location, traveling a distance of approximately $3.5 \mathrm{~km}$ and a gain of $2500 \mathrm{~m}$. At one point, the drone flew at an elevation of $8400 \mathrm{~m}$, well above the maximum service ceiling of $5000 \mathrm{~m}$ listed in the drone's specifications. ${ }^{3}$ Wind was approximately $40 \mathrm{~km} \cdot \mathrm{h}^{-1}$ and ambient air temperature $-10^{\circ} \mathrm{C}$; these conditions were expected to shorten the drone's flight capability from $27 \mathrm{~min}$ to approximately $18 \mathrm{~min}^{3}$ After a short aerial search and using the attached camera to scan the mountain slopes, they were able to visually locate the lost climber (Figure 1), confirm that he was still alive, and record his global positioning system (GPS) coordinates. Once the victim was located, the drone hovered until the victim acknowledged the presence of the drone with a wave. This reassured the victim that he had been located. Before being spotted by the drone, the victim had endured approximately 36 solitary hours at an elevation of around $7600 \mathrm{~m}^{4-6}$ This elevation is near the threshold of what alpinists call the "death zone," so named for its thin air and high mortality rates, ${ }^{7}$ underscoring the riskiness and urgency of the operation.

Once the victim was located, climbers equipped with adequate gear, food, water, and medication were quickly mobilized. Three climbers used the GPS coordinates from the drone to navigate the terrain from Broad Peak's Camp 3 to the victim's position. The rescue climbers were successful in assisting the victim off the steep slope and back down the mountain to Camp 3, where he was provided with limited supportive care. Impressively, the entire rescue effort was completed before dark on the same day the victim was located by the drone. Over the next $2 \mathrm{~d}$, the victim descended safely down to the mountain's base camp with the rescue group $(4850 \mathrm{~m}){ }^{5}$ His only reported injuries were superficial cuts and frostnip. Given the environment and extreme altitude, the climber was fortunate to have been located and assisted off the mountain with minimal complications. Without use of the drone, locating the climber and executing such an efficient rescue would have been unlikely.

\section{Discussion}

Drones have been successfully deployed to assist operations in a variety of fields, including industry, agriculture, geography, photography, and wildfire surveillance. ${ }^{2,7}$ However, literature is sparse when it comes to real-world applications of drones for SAR purposes. ${ }^{2}$ Although there have been efforts to apply drones in specific settings such as the delivery of medical equipment and supplies, ${ }^{8}$ to our knowledge, there are no previously reported cases for the use of drones during SAR operations conducted in difficult high-mountain terrain, generally considered to be altitudes over $4000 \mathrm{~m}$. The presented case demonstrates how drones can contribute to successful SAR operations in challenging environments, including, but certainly not limited to, high altitude.

The drone's real-time imagery and ability to record GPS coordinates can provide rescuers with valuable information regarding terrain, victim status, or possible

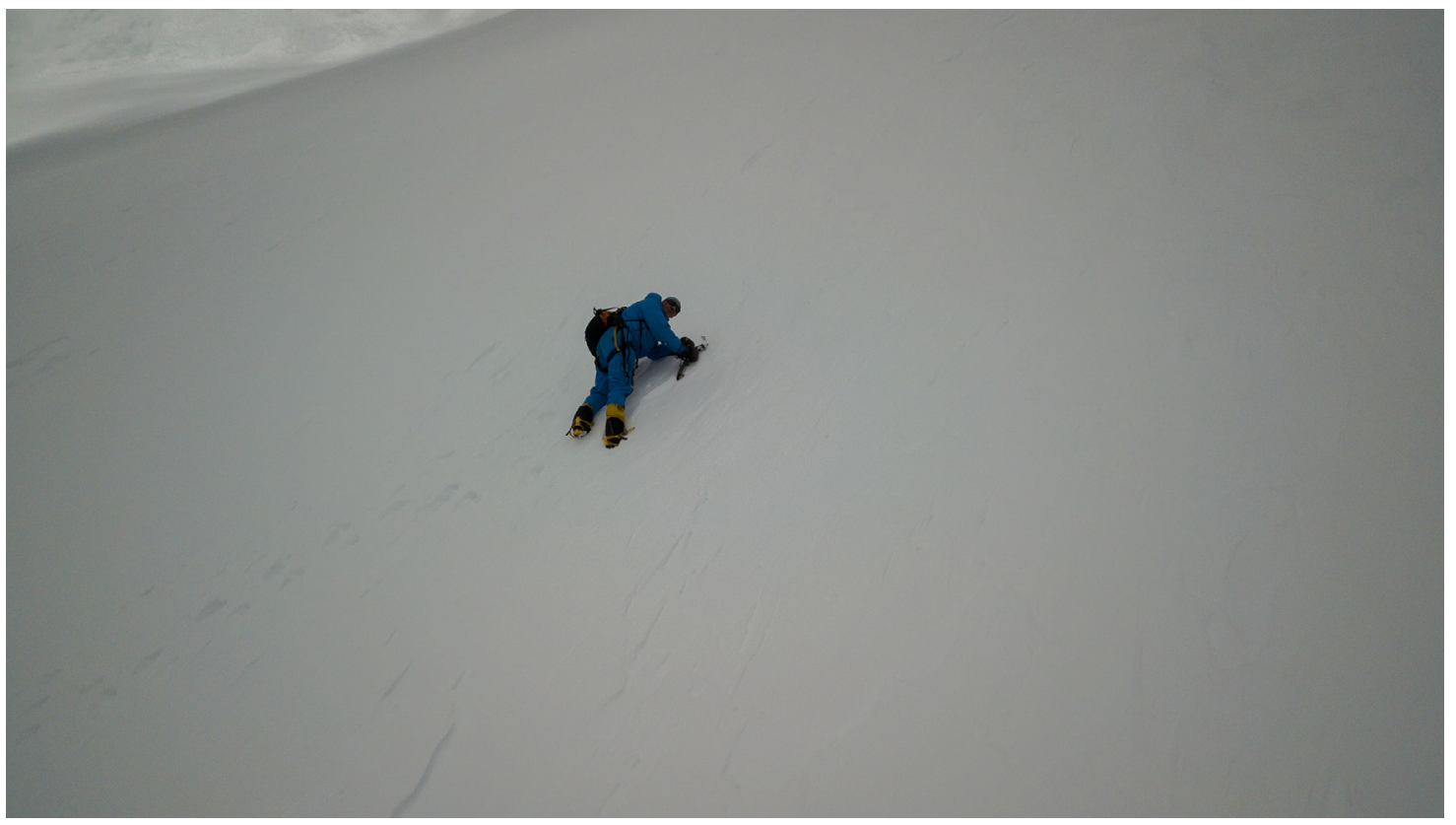

Figure 1. Victim identified using drone. 
hazards, which would allow for rescue teams to judiciously select gear, plan routes, and decide on appropriate personnel. Rescuers at high altitude put themselves at considerable risk when conducting SAR operations, and drone use to identify victim location and hazards has the potential to mitigate that risk.

This case adds to the limited but growing evidence that some consumer drones might be able to operate successfully at altitudes of over $8000 \mathrm{~m}$. As drone technology continues to advance, the range in potential uses for drones will also significantly expand. There is a great amount of promise in the future application of drones for SAR operations in all types of terrain and scenarios. This case adds to the immature but growing body of evidence supporting the effective use of drones in assisting SAR teams and operations in a variety of situations. ${ }^{2,9-12}$

Drones could be particularly effective in mitigating the challenges that SAR teams frequently confront: maintaining radio communications with incident command and SAR personnel, visually locating victims, delivering provisions and aid to victims, and guiding rescuers to facilitate rescuer-victim contact. ${ }^{8,10,13}$ One characteristic of consumer drones is that they are compact enough for rescuers to easily deploy in areas such as high altitude, canyons, and other difficult-to-access terrain. In addition to real-time use, drones may be used in the training of SAR teams and personnel during mock SAR events, providing valuable information that can be used to improve SAR procedures and protocols. ${ }^{11}$

Using a drone to aid in SAR has its potential advantages, but several limitations need to be considered. A drone requires additional training for SAR teams and forces them to be even more selective about the limited amount of gear they can take with them on a search. Teams should not rely too heavily on drones because they can be damaged, be lost, or malfunction while in operation. For many consumer drones, line of sight must be maintained with the pilot, making their use difficult in canyons or in places with tall trees. Battery life for consumer drones often provides less than an hour of continuous operation, limiting the amount of time SAR teams would be able to use the drone during a search. However, one study concluded that lithium "all-climate" batteries, such as those used in drones, can self-heat during operation, making them more resistant to the increased power loss induced by cold temperatures than conventional batteries. ${ }^{14}$ Lastly, there is a limit to the distance drones can travel while remaining under radio control of the pilot, possibly affecting SAR efforts in cases in which a significant amount of distance must be covered.

Although there are evident limitations for drone use during SAR operations, future studies could examine how to mitigate limitations such as battery life, navigation when line of sight is compromised (eg, in canyons), and range of operation. Future studies should investigate operation during inclement weather and federal aviation laws to determine how these might affect drone use for SAR; laws may differ from state to state or country to country. In the United States, for example, pilots must register their drones with the FAA, and there are specific legal restrictions on their use in terms of location, altitude, and maintaining line of sight with the pilot. ${ }^{15}$

With a cost of approximately US $\$ 1000,{ }^{3}$ SAR operation leaders should be made aware of the potential uses for drones in emergency situations, especially when faced with the cost of deploying full search parties. With proper training and experience, drones have the potential to become an invaluable tool to save the lives of victims whobecome lost in difficult terrain, victims experiencing medical emergencies in remote areas, and victims of natural disasters.

Acknowledgments: The authors acknowledge Andrew D. Nigh, MD, Brent Wright, and Alexis Horst for their helpful input and guidance in the production of this manuscript.

Author Contributions: Primary writer of the abstract and overall manuscript (JNM); main developer of the concept and idea of the project (JNM); consented to submission of the manuscript for publication (JNM, CJG, BMN, APH); significantly contributed to the writing and editing of the discussion and overall manuscript (CJG); prepared manuscript for publication (CJG); significantly contributed to the writing and background research of the introduction and case (BMN, APH); helped review and edit the manuscript in preparation for publication (BMN, APH).

Financial/Material Support: None.

Disclosures: None.

\section{References}

1. US Department of Transportation. FAA drone registry tops one million. Available at: https://www.transportation.gov/ briefing-room/faa-drone-registry-tops-one-million. Accessed February 11, 2019.

2. Karaca Y, Cicek M, Tatli O, Sahin A, Pasli S, Beser MF, et al. The potential use of unmanned aircraft systems (drones) in mountain search and rescue operations. Am J Emerg Med. 2018;36(4):583-8.

3. DJI user manual. Available at: https://www.dji.com/mavic/ info. Accessed February 11, 2019.

4. Kesteloo H. Mountaineer Rick Allen was feared dead on Broad Peak, but a DJI Mavic Pro drone found him alive. DroneDJ. Available at: https://dronedj.com/2018/07/16/ mountaineer-rick-allen-was-feared-dead-on-broad-peak-buta-dji-mavic-pro-drone-found-him-alive/. Accessed February $11,2019$.

5. McKenzie S. Drone spots Scots climber feared dead in Himalayas. BBC news. Available at: https://www.bbc.com/ news/uk-scotland-highlands-islands-44858758. Accessed February 11, 2019. 
6. Kesteloo H. Interview with Bartek Bargiel who used his DJI Mavic Pro to locate Rick Allen on Broad Peak [video]. DroneDJ. Available at: https://dronedj.com/2018/07/18/interviewwith-bartek-bargiel-who-used-his-dji-mavic-pro-to-locaterick-allen-on-broad-peak/. Accessed February 11, 2019.

7. Huey RB, Eguskitza X. Limits to human performance: elevated risks on high mountains. J Exp Bio. 2001;204(Pt 18):3115-9.

8. Zipline. We're providing $11,000,000$ people with instant access to urgent medicines. Available at: https://flyzipline. com/impact. Accessed February 28, 2019.

9. Van Tilburg C. First report of using portable unmanned aircraft systems (drones) for search and rescue. Wilderness Environ Med. 2017;28(2):116-8.

10. Claesson A, Fredman D, Svensson L, Ringh M, Hollenberg J, Nordberg P, et al. Unmanned aerial vehicles (drones) in out-of-hospital-cardiac-arrest. Scand J Trauma Resusc Emerg Med. 2016;24(1):124.
11. Abrahamsen HB. A remotely piloted aircraft system in major incident management: concept and pilot, feasibility study. BMC Emerg Med. 2015;15(1):1-12.

12. Amukele T, Ness PM, Tobian AA, Boyd J, Street J. Drone transportation of blood products. Transfusion. 2017;57 (3):582-8.

13. Claesson A, Svensson L, Nordberg P, Ringh M, Rosenqvist M, Djarv T, et al. Drones may be used to save lives in out of hospital cardiac arrest due to drowning. Resuscitation. 2017;114:152-6.

14. Wang CY, Zhang G, Ge S, Xu T, Ji Y, Yang XG, et al. Lithium-ion battery structue that self-heats at low temperatures. Nature. 2016;529(7587):515-8.

15. Federal Aviation Administration. Recreational fliers \& modeler community-based organizations. Available at: https:// www.faa.gov/uas/recreational_fliers. Accessed February 28, 2019. 